\section{Christoph Bachmann}

\section{Astaxanthin}

Astaxanthin ist eine natürliche Substanz aus der Stoffklasse der Carotinoide und gehört zur Untergruppe der Xanthophyllen, die sauerstoffhaltige Gruppen in Form von Hydroxy-, Carbonyl- oder Carboxy-Gruppen enthalten. Wie alle Carotinoide (Tab. 1) ist Astaxanthin ein Farbstoff und besitzt eine rötlich-violette Farbe. Die Substanz kommt vor allem in Grünalgen vor, ebenso in einigen Pilzen und Bakterien. Tiere, die Grünalgen in grösseren Mengen fressen, wie Lachse, Hummer, Krill oder auch Krabben, erhalten dadurch eine rötliche Färbung, ebenso Flamingos, die sich wiederum von diesen Tieren ernähren.

\section{Pharmakologische und klinische Eigenschaften}

Wie die meisten Carotinoide besitzt Astaxanthin antioxidative Eigenschaften und kann daher als Adjuvans und zur Prophylaxe bei verschiedenen Erkrankungen eingesetzt werden. Verschiedene In-vitro-Untersuchungen und pharmakologische Studien geben auch Hinweise auf antiphlogistische Eigenschaften [1]. Diese entzündungs-

Tab. 1. Einteilung der Carotinoide

\begin{tabular}{ll}
\hline Carotine & bestehen ausschliesslich aus \\
& Kohlenstoff und Wasserstoff; \\
& wichtiger Vertreter: \\
& Beta-Carotin \\
sauerstoffhaltige Derivate der \\
Canthophylle & Astaxanthin \\
\hline
\end{tabular}

\title{
Astaxanthin - ein neuer Wirkstoff für die Medizin?
}

Der folgende Beitrag stellt interessante pharmakologische und klinische Ergebnisse vor, die in entsprechenden Studien zum Carotinoid Astaxanthin gefunden wurden. Diese Substanz, ein starkes Antioxidans, könnte sich in naher Zukunft in der Medizin als neuer Wirkstoff zur Behandlung verschiedener Beschwerden anbieten.

hemmenden Eigenschaften scheinen auch bei Entzündungen an den Augen wirksam zu sein [2] sowie die Akkommodation zu verbessern [3].

Weiter gibt es auch Hinweise auf eine Wirksamkeit bei degenerativen Erkrankungen wie Arthritis und Arthrose [4].

Zudem gibt es Studien mit Sportlern, die gegenüber einer PlaceboGruppe für Astaxanthin einen Vorteil in der körperlichen Leistungsfähigkeit zeigen [5].

\section{Klinische Studien}

Bisher sind nur sehr wenige klinische Studien zu Astaxanthin publiziert worden. Es sollen hier aber zwei Studien vorgestellt werden, die sich einerseits mit den eingangs vorgestellten Eigenschaften von Astaxanthin und mit einer anderen, sehr interessanten möglichen Wirkung der Substanz befassen:

\section{Park et al., 2010: Immunantwort}

2010 publizierten Park et al. [6] eine randomisierte, doppelblinde, placebokontrollierte Studie, mit der sie entsprechende pharmakologische Resultate überprüften und die Wirksam- keit von Astaxanthin auf die Immunantwort, auf den oxidativen Stress und auf Entzündungsprozesse bei jungen Frauen untersuchten.

Dabei erhielten 21 gesunde, freiwillige Probandinnen mit einem Durchschnittsalter von 21,5 Jahren $(20,2-$ $22,8)$ während 8 Wochen täglich 0,2 oder 8 mg Astaxanthin. In der Woche 8 wurde ein Tuberkulin-Test durchgeführt.

\section{Resultate}

- Die Plasma-Konzentration nahm im Verlauf der Studie dosisabhängig zu. In den beiden AstaxanthinGruppen wurden folgende Resultate ermittelt:

- Astaxanthin verminderte nach 4 Wochen einen DNA-schädigenden Biomarker, ohne aber die Lipidperoxidation zu behindern.

- Bei den Probandinnen mit 2 mg Astaxanthin war nach 8 Wochen die Plasmakonzentration an C-reaktiven Proteinen signifikant niedriger $(\mathrm{p}<0,05)$.

- In den Astaxanthin-Gruppen wurden eine Stimulation der Mitogeninduzierten Lymphproliferation, ein Anstieg an zytotoxischer Aktivität von natürlichen Killerzellen sowie eine Erhöhung an T- und B-Zellen-Subpopulationen festgestellt.

\section{KARGER}

(C) 2015 S. Karger GmbH, Freiburg

Fax +497614520714 
- Astaxanthin veränderte jedoch nicht die Populationen von $\mathrm{T}_{\text {helper }}$, $\mathrm{T}_{\text {cytotxic }}$ - oder natürlichen Killerzellen.

- In der 2-mg-Gruppe wurde in Woche 8 ein höherer Prozentsatz an Leukozyten ermittelt, die LFA1-Marker produzierten.

- Die Gruppe mit 2 mg Astaxanthin wies eine höhere Tuberkulin-Antwort auf als die Placebo-Gruppe.

- Bei den Probandinnen mit 8 mg Astaxanthin wurde in Woche 8 kein Unterschied in der TNF (Tumornekrosefaktor)- und IL (Interleukin)-2-Plasmakonzentration, jedoch eine Plasmaerhöhung an IFN(Interferon)-Gamma und IL-6 festgestellt.

Aus diesen Resultaten schlossen die Autoren, dass die Gabe von Astaxanthin DNA-schädigende Biomarker und gewebeschädigende Akut-PhaseProteine vermindern und so sowohl die zellgesteuerte als auch die humoralbedingte Immunantwort bei jungen, gesunden Frauen stärkt.

\section{Comhaire et al., 2005: Fertilität}

Astaxantin wird in der Fischernährung verwendet und fördert dort offenbar die Fertilität der Fische. Comhaire et al. [7] untersuchten in einer doppelblinden, randomisierten, placebokontrollierten Studie, ob sich diese Wirksamkeit auch auf Menschen übertragen lässt.

Dabei erhielten 30 Männer mit einer seit mindestens 12 Monaten beste- henden Infertilität, deren Partnerinnen keine nachweisbare Infertilität aufwiesen, neben einer konventionellen, den WHO-Guidelines entsprechenden Behandlung während 3 Monaten entweder $16 \mathrm{mg} / \mathrm{Tag}$ Astaxanthin oder Placebo. Die Wirkung dieser Behandlung wurde anhand folgender Parameter gemessen:

Spermienparameter, Sauerstoffradikale (ROS), Spermien-Penetrationstest (zona-free hamster test), Serumhormone wie Testosteron, luteinisierendes Hormon (LH), follikelstimulierendes Hormon (FSH) sowie Inhibin B. Weiter wurden spontane oder Intrauterin-induzierte Schwangerschaften registriert.

\section{Resultate}

ROS und Inhibin B nahmen in der Astaxanthin-Gruppe $(n=11)$ gegenüber der Placebo-Gruppe $(n=19)$ signifikant ab. Die lineare Spermiengeschwindigkeit nahm in der Astaxanthin-Gruppe zu, nicht jedoch in der Placebo-Gruppe. Beim Spermien-Penetrationstest liess sich in der Astaxanthin-Gruppe gegenüber Placebo eine nicht signifikante Verbesserung feststellen.

Die gesamte sowie die pro Zyklus ermittelte Schwangerschaftsrate war in der Astaxanthin-Gruppe gegenüber Placebo signifikant höher $(\mathrm{p}=0,028$ bzw. p = 0,036) (Tab. 2).

Die Autoren der Studie folgerten aus diesen Resultaten vorsichtig: «Obwohl die vorliegende Studie eine positive Wirkung für Astaxanthin auf Spermienparameter und Fertilität zu
Tab. 2. Schwangerschaftsraten in Prozent

\begin{tabular}{lll}
\hline Gruppe & Gesamt (p) & Pro Zyklus (p) \\
\hline Astaxanthin & $54,5(0,028)$ & $23,1(0,036)$ \\
Placebo & 10,5 & 3,6 \\
\hline
\end{tabular}

haben scheint, müssen die ermittelten Resultate in einer breiter angelegten Studie bestätigt werden, bevor Astaxanthin zur zusätzlichen Behandlung von männlicher Infertilität empfohlen werden kann.»

\section{Fazit}

Verschiedene In-vitro-Untersuchungen, pharmakologische Studien und einige klinische Studien untersuchten die Eigenschaften des Xanthophyll-Carotinoids Astaxanthin. Dabei wurden zahlreiche Hinweise und zum Teil auch signifikante Resultate gefunden, dass diese Substanz als starkes Antioxidans eine positive Wirkung zur Verbesserung der Immunabwehr bei entzündlichen Prozessen, auch am Auge, einhergehend mit einer Verbesserung der Akkommodation, besitzt. Eine klinische Studie gibt Hinweise, dass Astaxanthin als $\mathrm{Zu}$ satzbehandlung eine positive Wirkung bei männlicher Infertilität besitzt.

Diese positiven Resultate geben Hoffnung, dass Astaxanthin in $\mathrm{Zu}$ kunft als neuer Wirkstoff in die Medizin eingeführt werden kann. Dazu braucht es noch weitere, breiter angelegte klinische Studien.

\section{Literatur}

1 Kurashige M, Okimasu E, Inoue Ml, Utsumi $\mathrm{K}$ : Inhibition of oxidative injury of biological membranes by astaxanthin. Physiol Chem Phys Med NMR 1990;22:27-38.

-2 Ohgami K, Shiratori K, Kotake S, Nishida T, Mizuki N, Yazawa K, Ohno S: Effects of astaxanthin on lipopolysaccharide-induced inflammation in vitro and in vivo. Invest Ophthalmol Vis Sci 2003;44:2694-2701.
3 Shiratori K, Ogami K, Nitta, T: The effects of astaxanthin on accommodation and asthenopia - efficacy identification study in healthy volunteers. Clin Med 2005;21:637-650.

4 Guerin M, Huntley M, Olaizola M: Haematococcus astaxanthin: applications for human health and nutrition. Trends Biotechnol 2003; 21:210-216.

5 Earnest CP, Lupo M, White KM, Church TS: Effect of astaxanthin on cycling time trial performance. Int J Sports Med 2011;18:73-88.
6 Park JS, Chyum JH, Kim YK, Line LL, Chew BO: Astaxanthin decreased oxidative stress and inflammation and enhanced immune response in humans. Nutr Metab (Lond) 2010; 7:18-28.

$\checkmark 7$ Comhaire FH, El Garem Y, Mahmoud A, Eertmans F, Schoonjans F: Combined conventional/antioxidant 'Astaxanthin' treatment for male infertility: a double blind, randomized trial. Asian J Androl 2005;7:257-262. 